\title{
Strength-duration relationship for extracellular neural stimulation
}

\author{
David Boinagrov ${ }^{1,2^{*}}$, Jim Loudin ${ }^{1,3}$, Daniel Palanker ${ }^{1,4}$ \\ From Nineteenth Annual Computational Neuroscience Meeting: CNS*2010 \\ San Antonio, TX, USA. 24-30 July 2010
}

Understanding the mechanisms and dynamics of extracellular neural stimulation is very important for the development of electro-neural interfaces in general, and for the design of stimulation waveforms and electrode configurations for neural prosthetic implants, in particular. The most common type of neural stimulation is extracellular, and yet its mechanisms and dynamics have scarcely been explored and described. Its strength-duration relationship is often assumed to be similar to the classical intracellular dependence, with a slope asymptotically approaching $1 / \tau$ at pulse durations $\tau$ shorter than chronaxy. The current study explores the basic mechanisms of extracellular stimulation and derives its strength-duration curve in a wide range of stimulus durations for various waveforms and cell shapes.

We used two different models of active membrane properties: the Hodgkin-Huxley model of the squid giant axon [1], and a six-channel salamander retinal ganglion cell model [2]. Three cell geometries were analyzed: an idealized planar cell with two uniformly polarized flat surfaces, and more realistic spherical and cylindrical shapes corresponding to the soma and unmyelinated axon or axon hillock.

The strength-duration relationship was found to differ significantly from classical intracellular models. For the Hodgkin-Huxley model at pulse durations between $4 \mu \mathrm{s}$ and $5 \mathrm{~ms}$ stimulation is dominated by sodium channels, and has a slope of approximately -0.72 in log-log coordinates. At shorter durations it is dominated by the potassium channels, and has a much lower slope of about -0.13 . With pulses shorter than cell polarization time (typically about $0.1-1 \mu \mathrm{s}$ ), it is dominated by polarization dynamics, and asymptotically approaches the classical -1 slope. For retinal ganglion cells we demonstrate that extracellular stimulation can have not only lower but also upper thresholds, and may be impossible below certain pulse durations. For both cell models we have found that in some stimulation regimes the stimulus can hyperpolarize cells, suppressing rather than stimulating spiking behavior. Thresholds for burst stimuli can be either higher or lower than that of a single pulse, depending on pulse duration. The modeled thresholds were found to be comparable to published experimental data obtained with rabbit retinal ganglion cells. These results provide a biophysical basis for understanding stimulation dynamics, and guidance for optimizing the efficacy and safety of extracellular neural stimulation.

\section{Author details}

${ }^{1}$ Hansen Experimental Physics Laboratory, Stanford University, Stanford, CA 94305, USA. ${ }^{2}$ Department of Physics, Stanford University, Stanford, CA 94305, USA. ${ }^{3}$ Department of Applied Physics, Stanford University, Stanford, CA 94305, USA. ${ }^{4}$ Department of Ophthalmology, Stanford University, Stanford, CA 94305, USA.

Published: 20 July 2010

\section{References}

1. Hodgkin AL, Huxley AF: A quantitative description of membrane current and its application to conduction and excitation in nerve. Journal of Physiology 1952, 117:500-554.

2. Fohlmeister JE, Miller RF: Impulse Encoding Mechanisms of Ganglion Cells in the Tiger Salamander Retina. Journal of Neurophysiology 1997, 78:1935-1947.

doi:10.1186/1471-2202-11-S1-P144

Cite this article as: Boinagrov et al:: Strength-duration relationship for extracellular neural stimulation. BMC Neuroscience 2010 11(Suppl 1):P144.

* Correspondence: boinagrov@gmail.com

${ }^{1}$ Hansen Experimental Physics Laboratory, Stanford University, Stanford,

CA 94305, USA 